Tony Frugier Carmen Cifuentes-Diaz Francesco D. Tiziano Judith Melki

\section{ADRESSE}

T. Frugier, C. Cifuentes-Diaz, F.D. Tiziano, J. Melki: Laboratoire de Neurogénétique Moléculaire, Inserm-Université d'Evry, EMI 9913, Génopole, CP 5724, 2, rue Gaston Crémieux, 91057 Évry Cedex, France. email : j.melki@genopole.inserm.fr

$\mathrm{m} / \mathrm{s} \mathrm{n}^{\circ} 6-7$, vol. 17 , juin-juillet 2001

\title{
Amyotrophies spinales: apport des modèles animaux à une meilleure compréhension de la physiopathologie et au développement des thérapeutiques
}

Les amyotrophies spinales sont des maladies neuromusculaires caractérisées par une dégénérescence des motoneurones associée à une paralysie et à une atrophie musculaires. L'identification du gène responsable ( $S M N)$, la connaissance des bases génétiques d'une forme sévère ou modérée de la maladie, une meilleure compréhension de la fonction de SM N et la production de modèles animaux ont permis de dégager plusieurs stratégies de recherches thérapeutiques. Celles-ci pourront être entreprises sur les modèles développés.

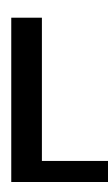

es amyotrophies spinales (ou SMA pour spinal muscular atrophy) représentent un groupe de maladies neuromusculaires caractérisées par une dégénérescence des motoneurones associée à une paralysie et à une atrophie musculaire.

\section{D}

\section{Des maladies cliniquement et génétiquement hétérogènes}

Il s'agit cliniquement d'affections hétérogènes par la distribution du déficit musculaire, prédominant aux racines des membres (les plus fréquentes) ou aux régions distales ou encore par l'atteinte ou le respect du muscle diaphragmatique (les plus fréquentes). Elles sont aussi hétérogènes du fait de l'âge d'apparition des symptômes qui va de la période néonatale à l'âge adulte (SMA de type I à IV). Enfin, tous les modes de transmission sont observés, autosomique récessif (les plus fréquentes), dominant ou encore récessif lié au chromosome $X$. Malgré cette impressionnante hétérogénéité, tant sur le plan clinique que génétique, le diagnostic est aisément posé devant un déficit musculaire symétrique, associé à une atrophie des masses musculaires, à une diminution, voire à une abolition des réflexes ostéotendineux sans atteinte faciale, ni d'atteinte des voies 
longues ou des fonctions cognitives [1]. L'absence d'élévation marquée des créatine kinases et de ralentissement des vitesses de conduction motrice ou sensitive sont très évocatrices du diagnostic d'amyotrophie spinale. Quant à la biopsie musculaire, dont l'indication est rarement posée depuis le développement de tests de diagnostic génétique, elle révèle une atrophie des fibres musculaires, isolées ou souvent regroupées en fascicules, associée à une redistribution du type histochimique ATPasique des fibres musculaires. Ces anomalies sont le témoin d'un processus de dénervation-réinnervation. Les amyotrophies spinales de l'enfant dont le déficit musculaire est à prédominance proximale et dont le mode transmission est autosomique récessif sont les plus fréquentes (incidence 1/ 6000 naissances) et nous concentrerons cet article de synthèse sur celles-ci [2-5].

\section{Bases génétiques: une situation singulière}

Par une approche désormais classique de clonage positionnel, SM N (pour survival of motor neuron ou SM N 1) a été identifié comme le gène dont les mutations sont responsables des amyotrophies spinales [6]. En effet, $93 \%$ des patients portent une délétion homozygote qui emporte au moins l'exon 7 et les patients restants portent une délétion hétérozygote, l'autre allèle portant une mutation non-sens ou faux-sens. Ce gène est dupliqué et une copie très homologue (SM N c ou SM N 2) est présente chez $95 \%$ de la population ordinaire [6]. SM N c est présent chez les malades. La question qui vient naturellement à l'esprit est de savoir pourquoi la délétion homozygote de SM N conduit au phénotype SMA alors que l'absence de gène $S M N C$ n'entraîne aucun phénotype apparent ? Le gène $S M N$ ne diffère de SM N c que par 5 substitutions nucléotidiques, dont trois siègent dans les introns, une dans l'exon 7 et la dernière dans l'exon 8 (non codant). La substitution nucléotidique de l'exon 7 ne modifie pas le sens du codon [6] (figure 1). L'analyse des transcrits dérivés de SM N et de SM NC a révélé la présence d'un épissage alternatif

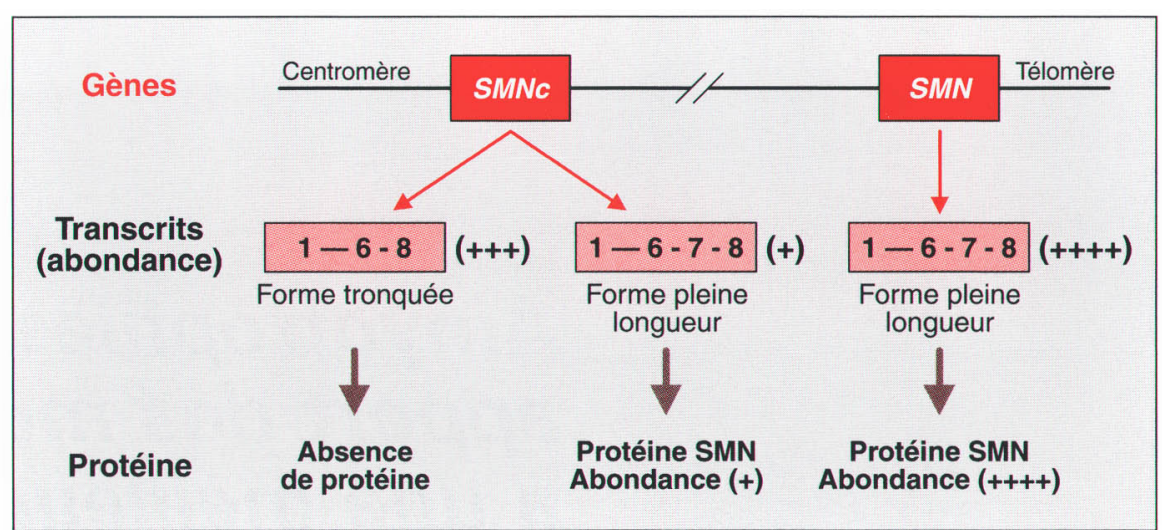

Figure 1. Caractéristiques du locus SMA, des transcrits et de la protéine SMN. Partie supérieure: organisation génomique du locus SMA. Les boîtes représentent les gènes. Partie intermédiaire: transcrits dérivés du gène SMN ou SMNc. Les numéros correspondent aux exons. Le nombre de croix indique l'abondance des transcrits.

qui conduit par conséquent à un transcrit tronqué pour l'exon 7 (la forme majoritaire) ou un transcrit pleine longueur (forme minoritaire) [6]. En revanche, le gène SM N est transcrit en une forme unique pleine longueur. II a été démontré que la substitution nucléotidique présente dans l'exon 7 de SMNc est le facteur déterminant dans la genèse de cet épissage alternatif [7]. La stabilité des transcrits pleine longueur ou tronqués est similaire [8]. Des expériences de transfection de plasmides portant I'ADNC de SM N pleine longueur ou tronqué avaient suggéré une instabilité de la protéine tronquée pour l'extrémité carboxy-terminale. Plus récemment, l'analyse de la protéine codée par le gène SM N murin délété pour l'exon 7 a révélé une diminution très marquée de la protéine correspondante indiquant un défaut de traduction ou une instabilité de la protéine SMN tronquée in vivo [9]. Par conséquent, le gène SM N c n'est que partiellement fonctionnel car seul le transcrit pleine longueur, en fait minoritaire, est traduit en une protéine stable, identique à celle codée par le gène SM N . Ainsi, le phénotype SMA semble dû à un effet de dosage de la protéine SMN. Cette constatation a été renforcée par l'observation d'une corrélation étroite entre le taux de protéine SMN et l'expression clinique de la maladie [10]. En effet, plus le niveau d'expression de SMN est faible, plus l'expression clinique est sévère. Cette corrélation étroite est sous-tendue par le nombre de gènes SM Nc chez les patients. En effet, le nombre de gènes SM Nc est variable tant dans la population ordinaire que chez les patients. Chez ces derniers, plus le nombre de gènes SM Nc est élevé, plus le phénotype clinique est modéré. Par l'analyse de l'ADN des patients en Southern blot ou par électrophorèse en champ pulsé (qui sépare les grands fragments d'ADN), un effet de dosage génique a été détecté dans les SMA de type I mais pas dans les types III. Dans les formes sévères de la maladie (SMA de type I), le gène SM N est délété à I'état homozygote, et le gène SM NC porté par chaque allèle reste présent; il en résulte une diminution du signal par rapport à un individu témoin. En revanche, dans les formes modérées de la maladie (SMA de type III), on ne détecte pas de diminution du signal génique malgré l'absence du gène $S M N$, ce qui suggère la survenue d'un événement de conversion génique remplaçant le gène SM $N$ par un gène SM N C $[6,11]$ (figure 2).

\section{SMN : une protéine, plusieurs fonctions}

Le gène $S M N$ code pour une protéine de 294 acides aminés qui ne présente aucune homologie avec les séquences contenues dans les bases de données. Il s'agit donc d'une nouvelle protéine. La première indication sur la fonction de SM N a été apportée par l'identification des partenaires de SMN dans les cellules 


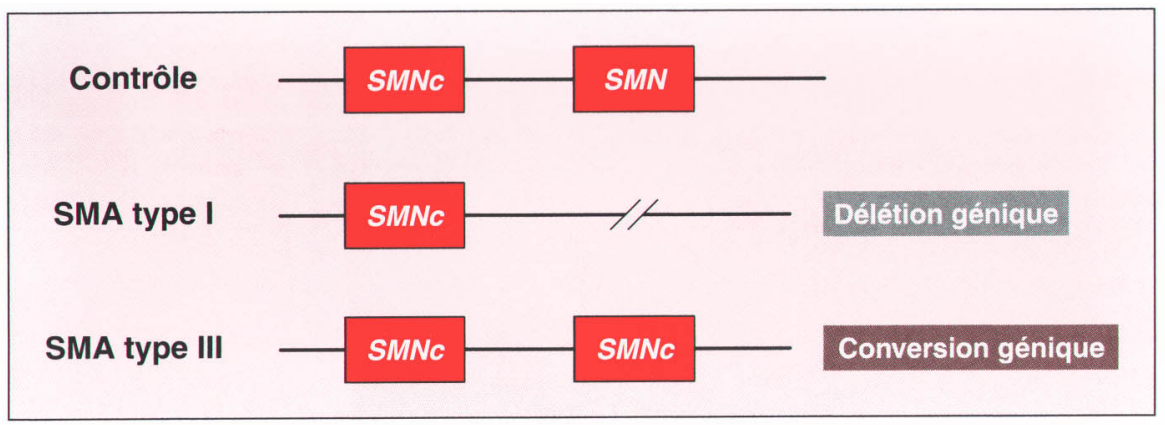

Figure 2. Bases génétiques des formes sévères ou modérées de SMA. Les boîtes représentent les gènes. Un seul allèle est représenté.

HeLa [12]. SMN interagit en effet avec les protéines du complexe splicéosomique, suggérant que SM N a un rôle important dans l'assemblage cytoplasmique des composants de ce complexe et dans la régénération de ces facteurs impliqués dans l'épissage des pré-ARN m [13-15]. SM N est localisée dans le cytoplasme mais aussi dans le noyau, concentrée dans des structures distinctes du nucléole et souvent associées à une autre structure nucléaire, les corps enroulés (coiled bodies). Cette structure nucléaire a été appelée gems, pour gemini of coiled bodies [12]. S'agit-il d'une nouvelle structure sub-nucléaire? Ce point reste discuté compte tenu de sa co-localisation très fréquente avec les coiled bodies. D'autres interactions protéiques directes ou indirectes avec SIP1 (SM N-interacting-protein 1 renommée par la suite Gemin 2), avec dp103 (Gemin 3) et RHA, une ARN hélicase, ont été plus récemment identifiées [16]. Cette protéine semble par conséquent impliquée dans des processus aussi variés mais aussi liés que la transcription, I'épissage ou encore le contrôle de la transcription [17].

Pourtant, la physiopathologie de cette affection reste à établir. Comment une protéine d'expression ubiquitaire peut-elle être responsable d'une maladie dégénérative restreinte au système neuromusculaire quand son niveau d'expression est abaissé ? Le système neuromusculaire est-il plus sensible à un effet de dosage de la protéine SMN et pourquoi? SMN aurait-elle une autre fonction ou une fonction plus spécialisée dans les tissus affectés par la mutation, via un partenaire ou une cible spécifique non encore identi- fiée ? L'identification des partenaires ou des cibles de SMN dans ces tissus devrait nous éclairer.

\section{La production de modèles animaux requiert des approches génétiques sophistiquées}

Pour tenter de répondre à ces questions, la production de modèles murins a été entreprise car aucune mutation spontanée du gène SM N murin n'était disponible. La première observation inattendue était I'absence de duplication de SM N chez la souris. L'inactivation comgène $\mathrm{SMN}$ murin. plète de SM N conduit à une létalité embryonnaire très précoce (au stade blastocyste) $[18,19]$. Si ces premiers résultats n'ont pas beaucoup éclairé sur la physiopathologie, ils ont renforcé néanmoins l'hypothèse selon laquelle SM N c constituait véritablement une «sauvegarde» partielle chez les patients SMA.

Deux stratégies assez sophistiquées ont été dès lors entreprises pour contourner la létalité embryonnaire et ont apporté des informations précieuses. L'une a consisté à reproduire une configuration génétique similaire à celle observée chez l'homme; la seconde, entreprise par notre groupe, a utilisé une approche de mutagenèse contrôlée. La stratégie adoptée par les groupes américano-allemands et taïwanais a consisté à exprimer par transgénèse le gène SMNC humain sur un fond génétique portant une délétion soit complète, soit limitée à l'exon $7 \mathrm{du}$ gène SM N $[20,21]$ (figure 3$)$. Selon le nombre de gènes SM N c intégrés dans le génome de la souris, le phénotype clinique est variable. Un nombre élevé conduit à un phénotype normal malgré l'inactivation de SM N. Un nombre faible est associé à un phénotype sévère caractérisé par une

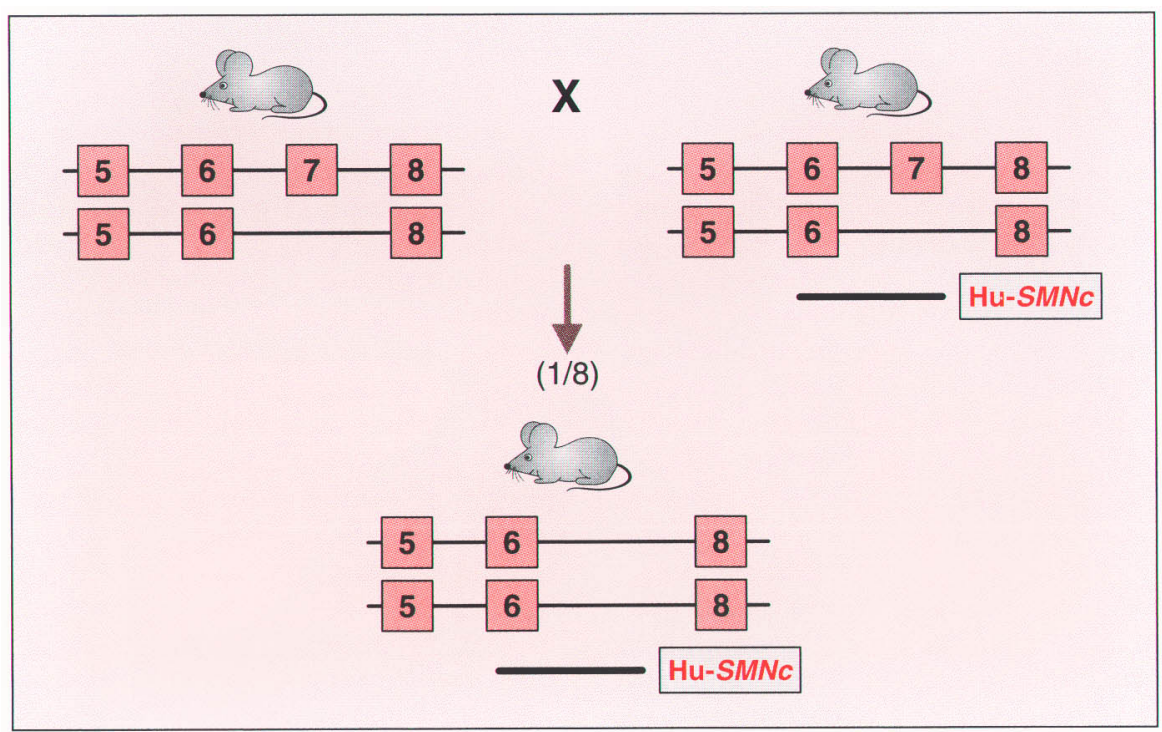

Figure 3. Première stratégie de production d'un modèle murin de SMA utilisant le gène SMNc humain. Cette stratégie combine une étape de recombinaison homologue conduisant à une délétion de l'exon 7 du gène SMN murin (boîte) et une étape de transgénèse utilisant le gène SMNc humain (trait plein). Un tel croisement aboutit dans $1 / 8$ des cas à des souris portant à la fois le transgène SMNc et la délétion homozygote de l'exon 7 du 
mortalité précoce des souris (de la naissance à 6 jours de vie), un déficit neuromusculaire caractérisé par une perte motoneuronale tardive et quelques signes extra-neurologiques inattendus (nécrose de membres ou de la queue) $[20,21]$. Cette stratégie a démontré que le gène SM Nc est capable de prévenir la survenue du phénotype SMA, dès lors que le nombre de copies est suffisant. II s'agit par conséquent d'un modèle de choix pour les stratégies thérapeutiques qui auraient pour but d'induire l'expression de SM Nc ou de moduler l'épissage alternatif de I'exon 7. En revanche, la mortalité précoce des animaux et la variabilité de l'expression phénotypique de la mutation au sein d'une même portée peuvent rendre difficile la manipulation de tels mutants.

La seconde stratégie développée par notre groupe a consisté à cibler la délétion de l'exon 7 du gène SM N , la plus fréquente des mutations observées chez les patients, dans les neurones ou le muscle squelettique [ 9 , $22,23]$. Elle a consisté à placer deux courtes séquences de 34 nucléotides (les séquences loxp) de part et d'autre de l'exon 7. La recombinase Cre, qui reconnaît spécifiquement ces séquences, va induire une délétion de I'ADN situé entre les séquences loxP, emportant ainsi l'exon 7 dès lors que ces séquences se trouvent dans la même orientation. Selon le lieu d'expression de la recombinase $\mathrm{Cre}$, la délétion de cette région pourra être ciblée à un tissu donné conduisant à une mosaïque tissulaire. Les tissus dans lesquels s'exprime la recombinase Cre porteront en effet la délétion de SM N, tandis que ceux dans lesquels la recombinase n'est pas exprimée ne porteront pas cette délétion (figure 4). En plaçant le gène de la recombinase Cre sous le contrôle du promoteur du gène de l'énolase spécifique des neurones, la délétion de cet exon a été dirigée dans les neurones, ce qui a permis de contourner la létalité embryonnaire. Le phénotype est caractérisé par une déficience musculaire progressive responsable d'une paralysie des mutants avec un âge de survie moyen de 28 jours [23]. Cette déficience motrice est associée à une dénervation musculaire très

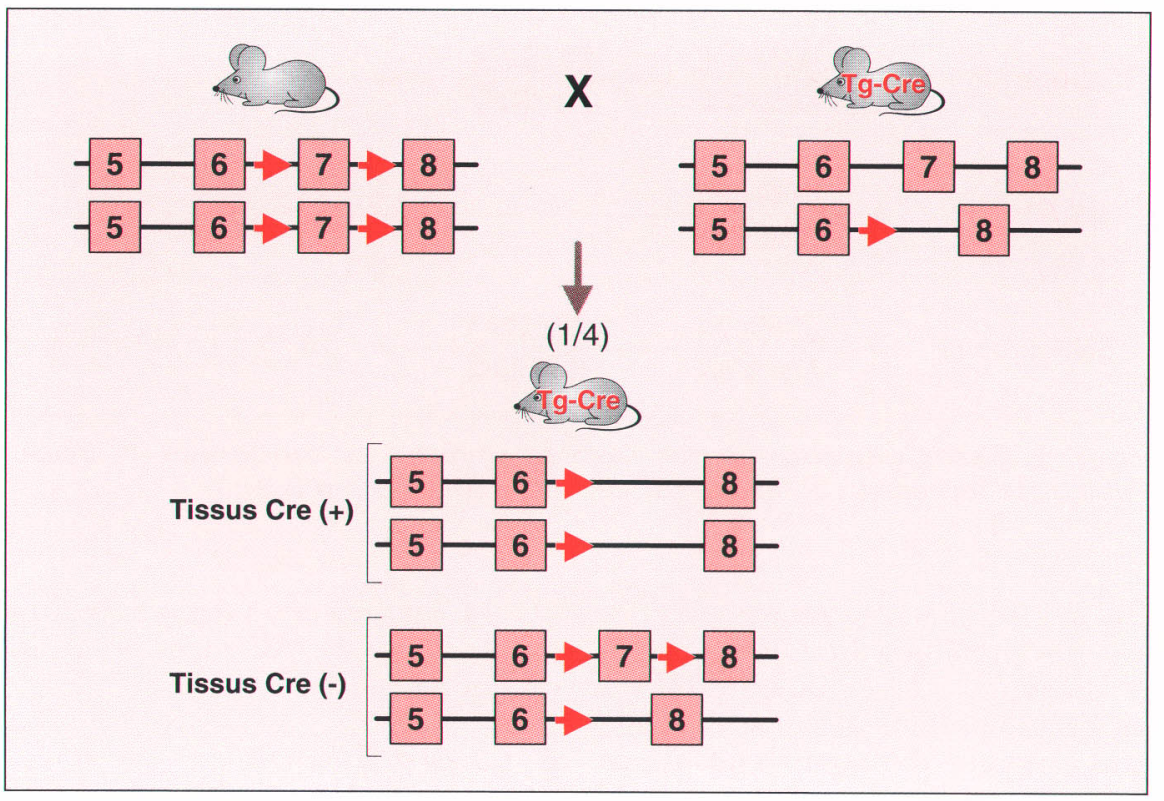

Figure 4. Seconde stratégie de production de modèles murins de SMA utilisant le système Cre-loxP. Par recombinaison homologue, deux séquences loxp ont été placées de part et d'autre de l'exon 7 (indiquées par des flèches, gauche). Une lignée transgénique a été établie exprimant la recombinase Cre dans un tissu donné (droite). Dans la descendance du croisement décrit, 1/4 des souris vont porter un génotype qui conduira, dans les tissus exprimant la recombinase Cre, à une délétion homozygote, alors que, dans les tissus ne l'exprimant pas, la délétion reste hétérozygote.

maladie humaine. Si le nombre de motoneurones des mutants semble voisin de la normale, des anomalies morphologiques ont été observées consistant en des plis de la membrane nucléaire, une déformation ou une position excentrée des noyaux, un aspect granulaire ou microvacuolaire du cytoplasme. Dans certains motoneurones, une fragmentation de la chromatine a été observée, suggérant la présence d'un processus apoptotique. Ces anomalies n'ont pas été jusqu'ici rapportées dans la maladie humaine et on peut supposer qu'il s'agit d'anomalies précédant la mort neuronale. Ces données ont permis de démontrer que les motoneurones sont des cellules cibles dans les SM A [23].

Pour déterminer si la délétion de SMN dans le muscle squelettique pouvait avoir un rôle dans la pathogénie des SMA, la même stratégie a été adoptée en ciblant cette fois-ci la même mutation dans le muscle squelettique. Le ciblage a été réalisé en plaçant la recombinase Cre sous le contrôle du promoteur du gène de I' $\alpha$-actine squelettique. De façon inat- tendue, les souris mâles ou femelles développent, après un intervalle libre de 3 semaines de vie, une paralysie musculaire progressive avec un âge de survie limité à 33 jours en moyenne [9]. L'analyse morphologique du muscle a révélé un aspect de dystrophie musculaire associant des images de nécrose, une inégalité de taille des fibres musculaires, une infiltration de cellules mononucléées dans le tissu interstitiel, et un processus de régénération avec des fibres musculaires à noyau centralisé. Aucune atteinte neurogène n'était associée, ce qui démontrait que l'atteinte musculaire était bien primitive et n'était pas responsable de la dégénérescence motoneuronale. Cette dystrophie musculaire caractérisée par un processus de nécroserégénération est associée à un défaut modéré de la perméabilité du sarcolemme avec élévation des taux de créatine kinases, une accumulation de bleu d'Evans, une diminution du niveau d'expression de la dystrophine et une élévation marquée du niveau d'expression de l'utrophine [9]. Un certain nombre d'éléments 
permettent la distinction entre la dystrophie musculaire associée à une mutation de SM N et celles dues à des mutations de la dystrophine ou des protéines associées chez la souris. La survie des mutants SMN est très limitée alors que les mutations de la dystrophine (souris mdx) ou des sarcoglycanes n'ont pas d'effet marqué sur la survie. Le processus de régénération musculaire, dont un témoin est la présence de petites fibres musculaires à noyau centralisé, est limité chez le mutant SMN ( $16 \%$ des fibres musculaires) alors qu'il est important chez les autres mutants (le pourcentage pouvant aller jusqu'à 70 à $80 \%$ des fibres musculaires). Les anomalies du sarcolemme du mutant SMN ont certaines particularités: les fibres présentant une accumulation du bleu d'Evans sont dispersées et peu fréquentes ( $5 \%$ ) alors que chez les mutants du complexe dystrophine, elles sont regroupées et plus fréquentes. Enfin, la diminution marquée du niveau d'expression de la dystrophine s'associe à un aspect en mosaïque du marquage du sarcolemme sans retentissement, ou sinon très modéré, sur les protéines associées à la dystrophine. Ainsi, ces résultats suggèrent-ils une atteinte musculaire primitive caractérisée par un processus sévère de dégénérescence musculaire secondaire à la mutation $d u$ gène $S M N$ dans le muscle squelettique et dont le mécanisme physiopathologique semble distinct des dystrophies musculaires liées à des mutations de la dystrophine ou des protéines associées [9]. Une atteinte primitive du muscle squelettique avait été préalablement suggérée par des études in vitro et semble désormais se confirmer in vivo $[24,25]$.

La mutation de SM N dirigée dans les neurones (mutant que nous appellerons neuronal) ou dans le muscle (mutant musculaire) ont des phéno-

Tableau I. Principales caractéristiques phénotypiques des modèles murins obtenus par délétion ciblée de l'exon 7 du gène SMN dans les neurones ou le muscle squelettique.

\section{Mutant neuronal}

Déficit moteur progressif

Dénervation musculaire Anomalies morphologiques des motoneurones types distincts (Tableau I). Le mutant neuronal est caractérisé par une paralysie musculaire due à un processus de dénervation associée à des anomalies morphologiques des motoneurones, alors que le mutant musculaire présente une dystrophie musculaire sans atteinte neurogène. Ces données suggèrent que, dans la maladie humaine, le déficit moteur pourrait associer un processus de dénervation musculaire et une dégénérescence musculaire primitive. Si les signes associés à la dénervation musculaire sont dans la grande majorité des cas au premier plan, il est important de noter qu'un quart des patients atteints de la forme modérée de la maladie (SM A type III ou maladie de Kugelberg Welander) présentent des signes compatibles avec un processus myopathique associant une élévation marquée des créatine kinases, une hypertrophie des mollets et des anomalies morphologiques du muscle évoquant un processus dystrophique (nécrose, infiltration monocytaire). Kugelberg et Welander avaient intitulé leur article original Heredofamilial juvenile muscular atrophy simulating muscular dystrophy [26] .

La mutation de SMN conduit à une atteinte dégénérative des neurones et du muscle squelettique, suggérant que les mécanismes physiopathologiques sont similaires. Par conséquent, le mutant musculaire pourra constituer un modèle approprié pour élucider ce mécanisme. Reste à savoir si ces atteintes sont spécifiques de ces tissus. La mutagenèse dirigée dans un autre tissu devrait permettre de répondre à cette question.

\section{Apports diagnostiques et stratégies thérapeutiques}

Quels ont été les apports de la génétique, d'une meilleure connaissance des bases moléculaires de l'expres-

\section{Mutant musculaire}

Déficit moteur progressif

Dystrophie musculaire

Absence d'atteinte neurogène

Déstabilisation du sarcolemme sion clinique de la maladie, de la fonction de SM N et des modèles animaux?

L'identification du gène $S M N$ a eu un impact médical majeur car elle permet désormais (1) de porter un diagnostic de SMA ou de l'exclure d'après un test génétique simple sans avoir recours à la biopsie musculaire; (2) de démembrer la nosologie des maladies du motoneurone dont l'expression clinique est atypique (s'associant à une arthrogrypose, à une atteinte diaphragmatique, à une atteinte musculaire à prédominance distale ou à une atteinte neurologique centrale); et (3) de proposer un conseil génétique éclairé aux parents ou individus apparentés. La facilité du diagnostic génétique aura dans le futur un impact important pour les essais thérapeutiques car une mutation du gène SM $N$ constituera un critère d'entrée, permettant par conséquent de regrouper une population homogène de patients. Le rôle de SMNC, véritable modulateur de l'expression clinique de la maladie, conduit naturellement à élaborer une première stratégie thérapeutique qui consisterait à essayer d'induire l'expression de SM Nc, en cherchant des molécules capables d'activer, de préférence sélectivement, le promoteur du gène SM NC $[8,27,28]$. L'élaboration de modèles cellulaires appropriés et le criblage à haut débit de molécules pour tenter de sélectionner de tels composants sont mis en place, et les modèles animaux, notamment ceux développés par le groupe d'A. Burghes et de $H$. Li, permettront de tester l'efficacité de ces molécules in vivo.

L'épissage alternatif de l'exon 7 du gène SM NC conduit à une protéine tronquée, instable, ou à une protéine pleine longueur dont le niveau d'expression est trop faible pour compenser le défaut de SM N chez les patients. Contrecarrer cet épissage alternatif est une stratégie élégante et un facteur impliqué dans l'épissage (de la famille des protéines SR) a révélé un tel effet in vitro [29]. De même, une stratégie fondée sur le criblage de molécules pour identifier celles ayant un tel effet est très séduisante. Comme dans la précédente stratégie, ces composants devront avoir une action sélective et efficace in vivo. 
Si SMN semble bien avoir un rôle dans l'épissage des pré-ARNm in vitro, la recherche de molécules capables de compenser le déficit de SM N est très attirante. Mais SMN a-telle ce rôle in vivo ? La recherche d'anomalies de l'épissage de transcrits extraits de la moelle épinière du mutant neuronal n'a révélé aucune anomalie significative, ce qui suggère que SMN n'est probablement pas un facteur d'épissage général. Si SMN a peut-être un rôle dans l'épissage de certains ARN, l'identification des ARN cibles reste à réaliser. Dans ce contexte, et aussi pour élucider la physiopathologie de cette affection, l'analyse comparée du transcriptome ou du protéome des tissus affectés - dérivés des modèles animaux (muscle squelettique et moelle épinière) - et des tissus non affectés peut être très informative en individualisant les molécules qui pourront constituer des cibles thérapeutiques.

Les modèles animaux ont apporté deux informations majeures: la mort motoneuronale semble être un processus tardif ou peu marqué et est, par conséquent, précédée par un dysfonctionnement. Cette observation ouvre une fenêtre thérapeutique consistant à tenter de restaurer la fonction ou de prévenir le dysfonctionnement soit de façon ciblée, en utilisant SMN, soit de façon non spécifique, en utilisant des facteurs ayant des propriétés neurotrophiques ou neuroprotectrices. Les agents pharmacologiques ayant de telles propriétés représentent de très bons candidats et les essais thérapeutiques peuvent être entrepris dès à présent sur les modèles développés. Un tel dysfonctionnement neuronal est-il réversible ? II s'agit naturellement d'une question majeure, qui conditionnera l'efficacité des stratégies thérapeutiques consistant à essayer d'induire l'expression de SM Nc, de contrecarrer l'épissage alternatif de SM N c ou d'essayer d'utiliser SM N comme «gène médicament». Les méthodes de transgénèse inductible devraient permettre de répondre à cette question. II s'agira alors, sur un modèle animal, d'induire l'expression d'un transgène SMN dont l'expression peut être contrôlée. De plus, cette stratégie permettra de pré- la maladie les lésions peuvent être réversibles ou stabilisées. Les modèles développés par notre groupe permettront de tester cette hypothèse. Enfin, cette stratégie permettra d'identifier les partenaires moléculaires associés au processus éventuel de réparation cellulaire ou tissulaire, par une analyse comparée des transcrits ou des protéines. Ces partenaires moléculaires constitueront des cibles thérapeutiques de choix.

La deuxième information apportée par l'analyse des modèles animaux est la probable atteinte musculaire et motoneuronale. L'atteinte combinée de ces tissus différents, mais si étroitement liés par la jonction neuromusculaire, jette un regard nouveau sur la pathogénie de cette maladie. Si le processus de dénervation musculaire est au premier plan, ne masquet-il pas une atteinte musculaire éventuelle ? Cette question pourra être abordée chez la souris, en ciblant la mutation à la fois dans le muscle squelettique et les neurones et en dressant le bilan des lésions musculaires. Ces données impliquent de considérer des approches thérapeutiques ciblant à la fois les neurones et le muscle squelettique ou encore des approches systémiques. Les approches pharmacologiques, déduites de la connaissance des mécanismes physiopathologiques à l'origine du processus dégénératif neuronal et musculaire, devront retenir par conséquent toute notre attention. Ces résultats conduisent aussi à envisager l'utilisation de cellules souches pluripotentes, dérivées par exemple de la moelle osseuse, et dont on connaît la capacité de s'engager dans la voie de différenciation neuronale ou musculaire $[30,31]$. Les mutants neuronaux et musculaires que nous avons développés seront des modèles de choix pour tester la colonisation du muscle squelettique ou du tissu neuronal par les cellules souches normales et apprécier l'effet bénéfique éventuel sur le phénotype SMA.

Une meilleure connaissance de la pathogénie de cette affection a permis d'ores et déjà de dégager des stratégies thérapeutiques rationnelles qui pourront être testées sur les modèles développés. $L a$ recherche physiopathologique et la recherche thérapeutique sont à l'évidence très étroitement liées

\section{Remerciements}

Les travaux réalisés par notre groupe ont bénéficié du soutien de I'Inserm, de l'Association française contre les myopathies, de la Fondation pour la recherche médicale, des associations Families of SMA (États-Unis), Andrew's Buddies (États-Unis) et de Génopôle. Nous remercions vivement Natacha Roblot, Emmanuelle Lacène, Vandana Joshi pour leur aide technique et Gaëlle Lefèvre pour l'aide à la rédaction du manuscrit.

\section{RÉFÉRENCES}

1. Munsat TL. Workshop report international SMA collaboration. Neuromusc Disord 1991; 1 : 81.

2. Roberts DF, Chavez J, Court SDM. The genetic component in child mortality. Arch Dis Child 1970; 45 : 33-8.

3. Pearn J. The gene frequency of acute Werdnig-H offmann disease (SM A type I). A total population survey in North-East England. J M ed Genet 1973; 10: 260- 5.

4. Pearn J. Incidence, prevalence and gene frequency studies of chronic childhood spinal muscular atrophy. I Med Genet 1978; 15: 409-13.

5. Czeizel A, Hamula J. A hungarian study on Werdnig-H offmann disease. J M ed Genet $1989 ; 26: 761-3$.

6. Lefebvre $S$, Bürglen L, Reboullet $S$, et al. Identification and characterization of a spinal muscular atrophy-determining gene. Cell 1995; 80: 155-65.

7. Lorson $\mathrm{CL}, \mathrm{H}$ ahnen $\mathrm{E}$, Androphy $\mathrm{EJ}$, Wirth B. A single nucleotide in the SMN gene regulates splicing and is responsible for spinal muscular atrophy. Proc Natl Acad Sci USA 1999; 96: 6307-11.

8. Baron-Delage S, Abadie A, EchanizLaguna $A$, Melki J, Beretta L. Interferons and IRF-1 induce expression of the survival motor neuron (SMN) genes. M ol M ed 2000; 6: $957-68$

9. Cifuentes-Diaz C, Frugier T, Tiziano FD, et al. Deletion of murine SM N exon 7 directed to skeletal muscle leads to severe muscular dystrophy. J Cell Biol 2001; 152: 110714 .

10. Lefebvre S, Burlet P, Liu Q, et al. Correlation between severity and SMN protein level in spinal muscular atrophy. $\mathrm{N}$ at $\mathrm{G}$ enet 1997 ; 16: 265-9.

11. Campbell L, Potter A, Ignatius J, Dubowitz V, Davies KE. Genomic variation and gene conversion in spinal muscular atrophy implications for disease process and clinical phenotype. Am J H um G en et 1997; 61: 40-50.

12. Liu Q, Dreyfuss G. A novel nuclear structure containing the survival of motor neuron proteins. EMBO J 1996; 15: 3555-65. 


\section{RÉFÉRENCES}

13. Liu Q, Fischer U, Wang F, Dreyfuss G. The spinal muscular atrophy disease gene product, SMN, and its associated protein SIP1 are in a complex with spliceosomal snRN P proteins. Cell 1997; 90 : 1013-21.

14. Fischer U, Liu Q, Dreyfuss G. The SMNSIP1 complex has an essential role in spliceosomal snRNP biogenesis. Cell 1997; 90 : 1023-9.

15. Pellizzoni L, Naoyuki K, Charroux B, Dreyfuss G. A novel function for SMN, the spinal muscular atrophy disease gene product, in pre-mRNA splicing. Cell 1998; $95: 615-24$.

16. Pellizzoni L, Charroux B, Rappsilber J, $M$ ann $M$, Dreyfuss $G$. A functional interaction between the survival motor neuron complex and RNA polymerase II. J Cell Biol 2001; 152: 75-86.

17. Strasswimmer J, Lorson $\mathrm{CL}$, Breiding $D E$, et al. Identification of survival motor neuron as a transcriptional activator-binding protein. H um M ol Genet 1999; 8: 121926.

18. Violet $L$, Bertrandy S, Bueno Brunialti $A L$, et al. CDNA isolation, expression and chromosomal localization of the mouse survival motor neuron gene (Smn). Genomics 1997; 40 : 185-8.

19. Schrank B, Götz R, Gunnersen JM, et al. Inactivation of the survival motor neuron gene, a candidate gene for human spina muscular atrophy, leads to massive cell death in early mouse embryos. Proc Natl A cad Sci USA 1997; 94 : 9920-5.

20. H sieh-Li HM, Chang JG, Jong YJ, et al. A mouse model for spinal muscular atrophy. Nat Genet $2000 ; 24: 66-70$.

21. Monani UR, Sendtner M, Coovert DD, et al. The human centromeric Survival Motor
Neuron gene (SMN2) rescues embryonic lethality in $\mathrm{SMN}^{-1-}$ mice and results in a mouse with spinal muscular atrophy. $\mathrm{Hum}$ $\mathrm{M}$ ol Genet 2000; 9: 333-9.

22. Sternberg N, Hamilton D. Bacteriophage P1 site- specific recombination. I. Recombination between IoxP sites. I Mol Biol 1981; 150: 467-86.

23. Frugier $T$, Tiziano FD, Cifuentes-Diaz $C$, et al. Nuclear targeting defect of SMN lacking the C-terminus in a mouse model of spinal muscular atrophy. Hum Mol Genet 2000; 9: 849-58

24. Henderson $\mathrm{CE}, \mathrm{H}$ auser $\mathrm{SL}, \mathrm{H}$ uchet $\mathrm{M}$, et al. Extracts of muscle biopsies from patients with spinal muscular atrophies inhibit neurite outgrowth from spinal neurons. Neurology 1987; 37: 1361-4.

25. Braun S, Croizat B, Lagrange MC, Warter JM, Poindron $P$. Constitutive muscular abnormalities in culture in spinal muscular atrophy. Lancet 1995; 345: 694-5.

26. Kugelberg E, Welander L. Heredofamilial juvenile muscular atrophy simulating muscular dystrophy. Acta Neurol Psychiatr 1956; 75 : 500-9.

27. Echaniz-Laguna A, Miniou $P$, Barthold $D$, Melki J. The promoters of the survival motor neuron gene (SMN) and its copy (SM Nc) share common regulatory elements. Am J H um G en et 1999; 64: 1365-70.

28. Monani UR, PcPherson JD, Burghes $\mathrm{AH} M$. Promoter analysis of the human centromeric and telomeric survival motor neuron genes (SMNC and SMNt). Biochim Biophys Ácta 1999; 1445 : 330-6.

29. Hofmann $Y$, Lorson $C L$, Stamm $S$, Androphy EJ, Wirth B. H tra2-beta 1 stimulates an exonic splicing enhancer and can restore full-length SMN expression to survival motor neuron 2 (SMN2). Proc Natl Acad Sci USA 2000; 97 : 9618-23.
30. Brazelton TR, Rossi FM, Keshet GI, Blau $H M$. From marrow to brain expression of neuronal phenotypes in adult mice. Science 2000; 290: 1775-9.

31. Ferrari G, Cusella-De Angelis G, Coletta $M$, et al. Muscle regeneration by bone marrow-derived myogenic progenitors. Science $1998 ; 279$ : 1528-30.

\section{Summary}

Animal models of spinal muscular atrophies: towards a better understanding of pathophysiology and development of therapeutics

Spinal muscular atrophies (SMA) are a group of neuromuscular disorders characterised by a degeneration of motorneurons associated with muscle paralysis and atrophy. The identification of the disease-causing gene, the understanding of the genetic bases of severe or mild forms of SMA, a better knowledge of SMN function and the generation of animal models made it possible to define several therapeutic strategies. These will be investigated on available models to test their efficiency.

\section{TIRÉS À PART}

J. Melki. 